\section{Who is who im TarMed}

\author{
Arbeitsgruppe TarMed FMH
}

$d r$. TarMed, ein Wort, das Interessierten im Gesundheitswesen zur Genüge bekannt ist, doch was genau ist TarMed, wer sind die beteiligten Parteien, wie funktionieren die Verhandlungen, Fragen, welche mit diesem kurzen Abriss beantwortet werden sollen.

\section{Die TarMed-Vertragsparteien}

Folgende Institutionen gelten im Rahmen des Projektes TarMed als Vertragsparteien:

- Konkordat Schweizerischer Krankenversicherer (KSK);

- Medizinaltarifkommission UVG (MTK), Militärversicherung (MV), Invalidenversicherung (IV);

- Verbindung Schweizer Ärztinnen und Ärzte (FMH);

- H+ Die Spitäler der Schweiz.

\section{Die TarMed-Vertragsparteien im einzelnen}

Kostenträger (Versicherer)

- KSK: Dachverband der Krankenversicherer in der Schweiz und damit Repräsentant des KVG-Bereiches.

- MTK, MV, IV: Kurz die «Eidgenossen», d.h. diejenigen Versicherer (staatlich oder öffentlich-rechtlich), welche im UVG-, MV- und IV-Bereich tätig sind.

Leistungserbringer

- FMH: Dachverband der Schweizer Ärztinnen und Ärzte.

- H+: Dachverband der Spitäler der Schweiz.

Beobachterstatus

- Schweizerische Sanitätsdirektorenkonferenz (SDK)

\section{Die TarMed-Gremien}

\section{POL TarMed}

Heisst soviel wie Projektoberleitung, in welcher die Spitzen der Vertragsparteien (in concreto: Dr. P. Saladin [H+], Dr. W. Morger [MTK], Herr M. Manser [KSK] und Dr. H. H. Brunner [FMH]) Einsitz nehmen. Dieses Gremium ist vergleichbar mit einem Verwaltungsrat, welcher letztlich die Verantwortung für das gesamte Projekt trägt.

\section{PL TarMed}

Projektleiter TarMed, also diejenigen von den Vertragsparteien delegierten Personen, welche die effektiven Verhandlungen führen. In der PL TarMed ist zusätzlich die SDK im Beobachterstatus vertreten.

\section{Sekretariat TarMed}

Wird durch die Zentralstelle für Medizinaltarife (ZMT) der Suva geführt.

\section{TarMed, die Welt der bilateralen Verträge}

Sowohl im UVG- also auch im KVG-Bereich werden bilaterale Verträge zwischen den Kostenträgern (Versicherern) und den Leistungserbringern (Ärzteschaft, Spitäler) abgeschlossen.

\section{UVG-Bereich}

\section{Versicherer - Ärzteschaft}

(in concreto: $M T K / M V / I V-F M H$ )

Die «Eidgenossen" auf Versicherer- und Ärzteseite schliessen zusammen den Tarifvertrag TarMed gemäss den gesetzlichen Vorgaben des UVG, des IVG, des MVG sowie deren einschlägigen Verordnungen ab. In diesem Vertrag werden die Regelungen betreffend der von selbständig praktizierenden Ärzten im Rahmen des TarMed erbrachten Leistungen vereinbart.

\section{Versicherer - Spitäler}

(in concreto: $M T K / M V / I V-H+$ )

Analog dem Vertrag Versicherer-Ärzteschaft wird ein Vertrag zwischen Versicherer und H+ abgeschlossen. Dieser Vertrag hält die Bestimmungen betreffend der von den angestellten Ärzten im Rahmen des TarMed erbrachten Leistungen fest.

\section{KVG-Bereich}

Versicherer - Ärzteschaft (in concreto: KSK - FMH) Da der Vollzug des KVG in die Hoheit der Kantone fällt, können KSK und FMH auf eidgenössischer Ebene lediglich einen Rahmenvertrag aushandeln. Wie es der Name bereits sagt, steckt dieser Vertrag den Rahmen für die Verhandlungen in den einzelnen Kantonen ab. Mit anderen Worten: Die kantonalen Verträge zwischen den kantonalen Krankenkassenkonkordaten und den kantonalen Ärztegesellschaften bezüglich der Bestimmungen für die Leistungserbringung von selbständig praktizierenden Ärzte müssen den Anforderungen des Rahmenvertrages genügen bzw. dürfen diesem nicht widersprechen.

\section{Versicherer - Spitäler (in concreto: $K S K-H+$ )}

Analog dem Vertrag KSK-FMH wird ein Vertrag zwischen KSK und H+ abgeschlossen. Dieser Vertrag hält die Bestimmungen betreffend der von den angestellten Ärzten im Rahmen des TarMed erbrachten Leistungen fest. 


\section{Die Nachfolgeorganisation (NFO)}

Mit Einführung des TarMed im UVG- und KVG-Bereich ist das eigentliche Projekt abgeschlossen. Das Tarifwerk muss aber weiterhin im Sinne von Unterhaltsarbeiten gepflegt werden. Zu diesem Zweck wird ein Nachfolgeorganisation ins Leben gerufen, welcher wiederum die Delegierten der Vertragsparteien angehören.

\section{Die TarMed-Delegationen der FMH}

Um die vielfältigen Aufgaben rund um TarMed zu bewältigen, stehen die verschiedensten Personen im Einsatz:

- Zentralvorstand der FMH: Oberaufsicht;

- Präsident FMH: Delegationsleiter bei den UVGVerhandlungen; Mitglied der Delegation in den KVG-Verhandlungen, Mitglied der POL;

- Präsident G7: Der Ausschuss der kantonalen Ärztegesellschaften, G7 genannt, führt im wesentlichen die Verhandlungen im KVG-Bereich;

- Leiter Tarifdienst: Projektleiter TarMed, Mitglied der TaKo;

- TaKo: Tarifkommission FMH; Stabsstelle des Projektleiters TarMed. 